\title{
MIDLUTEAL COLOUR DOPPLER ULTRASONOGRAPHIC ASSESSMENT OF UTERINE BLOOD FLOW IN UNEXPLAINED INFERTILITY AND RECURRENT MISCARRIAGE
}

\author{
Wafaa M Aboul Enien, M.D., Amal Z. Azzam, M.D.
}

Department of Obstetrics \& Gynaecology, Faculty of Medicine, Alexandria University.

\section{ABSTRACT}

Objective: To evaluate the difference in uterine artery impedance to blood flow among women with unexplained infertility and recurrent pregnancy loss in the midluteal phase of spontancous cycles.

Design: Prospective study.

Setting: Department of Obstetrics and Gynaecology, Shatby University Hospital, Alexandria.

Subjects: Thirty women with unexplained infertility (group A), twenty women with a history of recurrent pregnancy loss (group B) and twenty fertile controls (group C).

Interventions: Midluteal sonographic evaluation of uterine artery impedance to blood flow and endometrial thickness. Main outcome measures: The mean right and left uterine artery pulsatility index (PI values).

Results: PI values did not differ significantly in unexplained infertility ( $1.83 \pm 0.55$ ) and recurrent pregnancy loss (1.73 $\pm 0.49)$ compared to controls $(1.84 \pm 0.48)$. However, endometrial thickness was significantly decreased in unexplained infertility $(9.17 \pm 1.93 \mathrm{~mm})$ and recurrent pregnancy loss $(9.1 \pm 2.13 \mathrm{~mm})$ compared 10 controls (12.6 \pm $2.22 \mathrm{~mm}$ ). There was no significant correlation between PI values and endometrial thickness in all the studied groups $(p>0.05)$. However there was a significant positive correlation between PI values and age $(r=0.4666, p=0.001)$.

Conclusion: Evaluation of uterine impedance by pulsed Doppler ultrasound in patients with unexplained infertility and recurrent miscarriage is considered an investigational technique without sufficient proven clinical benefits.

Keywords: Midluteal, Doppler, unexplained infertility, recurrent pregnancy loss, pulsatility index, endometrial thickness.

\section{INTRODUCTION}

Recurrent pregnancy loss may be a consequence of an abnormal embryonic karyotype, or to anatomic, endocrinological, immunological, microbiological or maternal factors affecting the endometrium. The various methods used in studying the endometrium include morphological studies, immunohistochemistry, measurement of endometrial proteins in plasma and uterine flushings, cytokine expression in endometrial cells, leukocyte populations in the endometrium and ultrasonographic and hysteroscopic studies. However, vascular changes associated with the pathology of recurrent pregnancy loss remain poorly studied ${ }^{(1,2,3)}$

Uterine receptivity is regulated by a number of factors including uterine perfusion and is of great importance in achieving a normal pregnancy. ${ }^{(4)}$ The uterine arteries are the most important vessels providing blood to the uterus. Good uterine perfusion, combined with the rapid growth of the

Corresponding author : Dr. Wafaa Aboul Enien, Department of Obstetrics \& Gynaecology, Shatby University Hospital, Alexandria 
coiled arterioles and abundant angiogenesis in the luteal phase, reflect the preparation and maintenance of a suitable environment for embryo implantation during each menstrual cycle, ${ }^{(5)}$

Initially, pulsed Doppler imaging in combination with transabdominal B mode imaging was used to test the ovarian and uterine vasculature. ${ }^{(6)}$ Performing Doppler ultrasound using a vaginal probe has several advantages compared to an abdominal transducer. The proximity of the transducer to the pelvic organs means that higher frequencies can be used, thus providing an image with better resolution. Moreover, there are no subcutaneous tissues interfaced between the transducer and the pelvic organs, hence artifacts from multiple reflections is reduced. The empty bladder also gives more patient comfort and convenience. ${ }^{(7)}$

By using color Doppler, the uterine artery is more easily visualized lateral to the internal os and as the Doppler beam is insonating at a low angle, a large Doppler frequency shift is obtained, which reduces the possibility of error when impedance indices are measured. ${ }^{(8)}$

Transvaginal color Doppler ultrasound has been used to demonstrate that in normal fertile non-pregnant women, uterine arterial impedance has its lowest value during the midluteal phase of the menstrual cycle. ${ }^{(9)}$ This reflects optimal uterine vascularization during the implantation of the human blastocyst. It is however unknown whether the midluteal uterine impedance is different in patients with unexplained infertility or recurrent pregnancy loss.

During fertility investigation and treatment, an assessment of endometrial receptivity is generally based on endometrial thickness and appearance in conjunction with an estimation of uterine artery blood flow velocities using Doppler ultrasound. ${ }^{(10)}$ A typical trilaminar appearance with a minimum thickness of $7 \mathrm{~mm}$ and a uterine artery pulsatility index of $<3.0$ are regarded as sound markers of endometrial receptivity. (11) Patients who have an abnormal endometrial receptivity pattern during their natural cycle may in fact constitute a proportion of patients previously described as having unexplained infertility. Identifying those patients prior to treatment gives an opportunity to consider therapy to improve uterine and endometrial blood flow and subsequently endometrial receptivity. ${ }^{(12)}$

The aim of the present study was to analyze the uterine flow velocity waveforms in the midluteal phase of unstimulated non-conceptional cycles in women with unexplained infertility and recurrent pregnancy loss compared to fertile controls.

\section{MATERIALS \& METHODS}

Seventy women aged 20-35 years attending the Gynaecology Outpatient Clinic at EL-Shatby University Hospital, Alexandria were recruited for the study. Patients were categorized into:group (A) which included thirty women with unexplained infertility, group (B) included twenty women with a history of recurrent pregnancy loss (RPL) and group (C) included twenty non pregnant fertile controls with no history of reproductive failure. Unexplained infertility was diagnosed on the basis of a failure to conceive after 2 years of unprotected intercourse in women who had regular menstrual cycles with serum progesterone in the midluteal phase $>9.5 \mathrm{ng} / \mathrm{ml}(30 \mathrm{nmol} / \mathrm{L})$, a normal pelvis on laparoscopy, and whose partners had normal seminal analyses. ${ }^{(13)}$

Recurrent pregnancy loss was defined as three or more sequential spontaneous pregnancy losses 
fathered by the same partner. Exclusion criteria included women with uterine anomalies, impaired glucose tolerance, abnormal thyroid function or anti-phospholipid antibodies.

An informed consent was obtained from each patient prior to examination.

\section{Vaginal ultrasound and colour flow imaging}

Transvaginal ultrasonography associated with Doppler flow measurements was performed in the midluteal phase of a spontaneous non-pregnant cycle. Blood flow evaluations were performed in the morning to avoid fluctuations due to circadian rhythm of uterine artery blood flow. ${ }^{(14)}$ All women rested in a waiting room for more than twenty minutes before being scanned to minimize any possible effects of exercise on uterine blood flow. All scans were performed by the same operator.

For flow visualization, color Doppler was used. The pulsed Doppler signals were obtained using the $2 \mathrm{~mm}$ volume cursor. A scanner (Model EUB-515, Hitachi Medical Corporation, Tokyo, Japan) was used with a $6.5 \mathrm{MHz}$ transvaginal probe for imaging and $2.5 \mathrm{MHz}$ pulsed Doppler system for blood flow analysis. The intensity of the colour produced was proportionate to the magnitude of the Doppler frequency shift. The configuration used was a red colour indicating blood flow towards, and a blue colour denoting blood flow away from the probe. ${ }^{(15)}$

The uterus was examined systematically and the maximum thickness of the endometrium was measured as the maximum distance between each myometrial-endometrial interface through the central longitudinal axis of the uterus. ${ }^{(16)}$ The probe was directed in the right vaginal fornix to identify the ascending branch of the uterine artery and the flow velocity waveforms were recorded on videotape for subsequent analysis. The probe was then moved to the left fornix to identify the left uterine artery. The pulsatility index (PI) was determined for each uterine artery. It is a useful way of expressing blood flow impedance distal to the point of sampling. (17)

The PI was calculated as the difference between the maximum systolic blood flow and minimal diastolic blood flow divided by the mean flow throughout the cycle. In this formula, PI increases if the proximal flow remains constant while the distal vascular bed constricts. Conversely, a low PI value indicates decreased impedance to blood flow in the distal vasculature. The average PI of the bilateral uterine arteries (the ascending branch) was calculated.

\section{Statistical analysis}

Data are presented as mean $\pm S D$. The data were tested by F-test (ANOVA) to compare arithmetic means between studied groups. Subsequent statistical analysis was performed using the Mann-Whitney test or Pearson's correlation coefficient as appropriate. $\mathrm{P}$ value $<0.05$ was considered statistically significant.

\section{RESULTS}

Seventy women were included in the study. The mean women's age did not differ significantly between the three studied groups $(P=0.43)$.

Both uterine arteries were clearly visualized in all patients using the transvaginal transducer and the Doppler signals were obtained and averaged. There was no significant difference in the PI values between the right and left uterine arteries.

The PI values of the uterine artery did not differ significantly between groups. (Table I). However, the endometrial thickness was significantly 
decreased in groups $\mathrm{A}$ and $\mathrm{B}$ compared to controls. There was no significant difference between groups A and B (Table I). The uterine artery PI values were positively correlated with age but did not correlate with endometrial thickness in all the studied groups (Table II).

Figure 1, 2, and 3 illustrate a flow velocity waveform during the midluteal phase of a spontaneous cycle in the three studied groups.

\section{DISCUSSION}

The introduction of pulsed Doppler ultrasonography has provided non invasive means for evaluation of flow velocity waveforms from uterine arteries at any time during the menstrual cycle. ${ }^{(18)}$ There is a relation between the concentration of ovarian hormones in peripheral venous plasma and uterine artery blood flow parameters. ${ }^{(7,9)}$ Studies on flow waveforms of uterine artery during the normal menstrual cycle show a sharp increase in end-diastolic velocities between the proliferative and secretory phases of the cycle. However, the lowest flow impedance is recognized during the time of peak luteal function (mid-luteal phase) during which implantation is most likely to occur. ${ }^{(19)}$ Hence, measurements of PI in the uterine arteries in the midluteal phase might isolate patients with impaired uterine perfusion.

In the current study, there was no significant difference between the PI values of the right and left uterine arteries. Similar results were observed by others. ${ }^{(15)}$ However, Tan et al ${ }^{(8)}$ reported that the uterine blood flow velocity was higher and PI value lower in the dominant uterine artery compared to the contralateral side during the midluteal phase. This was attributed to the high metabolic activity of the corpus luteum and its surrounding stroma which may directly influence uterine artery vascular resistance by a local effect of the metabolic and vasoactive products. Furthermore, since the ovary derives a proportion of its blood supply from the tubal branch of the uterine artery, if its resistance is primarily low because of the corpus luteum, the ovary may act as a low resistance shunt causing further decline in impedance to blood flow in the ipsilateral uterine artery.

In the present study, there was no significant difference in the PI values in patients with unexplained infertility compared to controls. This is in agreement with previous investigators $(7,18,20)$ who demonstrated no difference in uterine and ovarian blood flow impendance between fertile and infertile patients in the midluteal phase. On the contrary, others ${ }^{(15,21,22)}$ reported increased uterine artery impedance in unexplained infertility, and attributed that to attenuation in uterine response to circulating ovarian hormones.

In the current study, the PI values in women with recurrent miscarriage did not differ significantly compared to controls. Similar results were reported by Jirous et $\mathrm{al}^{(23)}$ who did not confirm any significant difference in the midluteal Doppler indices characterizing uterine and intraovarian blood flow in women with recurrent miscarriage. In contrast, Habara et $\mathrm{al}^{(24)}$ and Nakatsuka et $\mathrm{al}^{(25)}$ reported elevated uterine arterial impendance among women with recurrent miscarriage and attributed that to abnormality in uterine perfusion during the midluteal phase of non-conceptional cycles.

The endometrium has an exceptional capacity to undergo changes in structure and function during the menstrual cycle. Increased endometrial vascularity depends on changes in uterine arcuate and radial artery blood flow. Resistance to flow which is inversely correlated to tissue perfusion drops towards ovulation and during the luteal phase, ${ }^{(26)}$ 
In the current study, endometrial thickness was significantly decreased in unexplained infertility and recurrent miscarriage compared to controls. This might be attributed to suboptimal endometrial perfusion. Similar observations were reported in previous studies. ${ }^{(6,15,24,27)}$ Moreover, in a recent study by Check et al ${ }^{(28)}$, it has been demonstrated that failure to develop a homogenous hyperechogenic sonographic endometrial echo pattern in the midluteal phase was associated with decreased fecundity in infertile women not receiving follicle-maturing drugs.

In our study, there was no significant correlation between PI and endometrial thickness in all studied groups. Similar results were obtained by Habara et $\mathrm{al}^{(24)}$ in women with recurrent pregnancy loss. Moreover, negative reports concerning the correlation between PI and endometrial thickness were reported in women undergoing IVF-embryo transfer. ${ }^{(29,30,31)}$

However, Steer et $\mathrm{al}^{(11)}$ reported an inverse correlation between uterine artery impedance and uterine receptivity in IVF cycles. Similar findings were reported by previous investigators. ${ }^{(7,15,26)}$ Moreover, Coulam et al ${ }^{(32)}$ reported that a PI $>3$ and an endometrial thickness $<6 \mathrm{~mm}$ were poor prognostic factors for embryo implantation in IVF cycles.

Finally, a significant correlation was found in our study between uterine artery PI and age. It has been known that fertility in women declines with age. ${ }^{(30)}$ Yaron et al ${ }^{(33)}$ reported that a decline in endometrial receptivity, which is associated with a decrease in uterine perfusion, may play an important role in the decrease of implantation rates with age. On the contrary, Habara et $\mathrm{al}^{(24)}$ and Goswamy et $\mathrm{al}^{(16)}$ did not find age-related elevation in uterine arterial PI, suggesting that ageing affects uterine perfusion less than other factors.

The results of the present study were predominantly negative. Too much overlap occurred between the studied groups as regards the uterine PI values rendering it not a clinically useful predictor. Endometrial thickness and hence subendometrial perfusion might be more accurate than uterine perfusion and more likely to become a vital tool in the investigation of those patients. This might be amenable to treatment by perfusion enhancers.

In conclusion, the use of pulsed Doppler ultrasound in the evaluation of uterine impedance in women with unexplained infertility and recurrent miscarriage is still considered an investigational technique without sufficient proven clinical benefit.

\section{REFERENCES}

1- Kattch WH. Recurrent pregnancy loss: an update. Curr Opin Obstet Gynecol 1999; 11:435-439.

2- Li TC. Recurrent miscarriage: principles of management. Hum Reprod 1998; 13: 478-482.

3- Li TC, Tuckerman EM, Laird SM. Endometrial factors in recurrent miscarriage. Hum Reprod Update 2002; 8(1): 43-52.

4- Seppala M. Factors of importance for implantation. In: Seppala M, ed. Bailliere's Clinical Obstetrics and Gynaecology. London Baillicre Tindall, 1991

5- Kuo HC, Hsu CC, Wang ST, Huang KE. Aspirin improves uterine blood flow in the peri-implantation period. J Formos Med Assoc 1997; 96: 253-257.

6- Goswamy RK, Steptoe PC. Doppler ultrasound studies of the uterine artery in spontaneous ovarian cycles. Hum Reprod 1988; 3: 721-726.

7- Kurjak A, Kupesic-Urek S, Schulman H, Zalud I. Transvaginal color flow Doppler in the assessment of ovarian and uterine blood flow in infertile women. Fertil Steril 1991; 56: 870-873.

8- Tan S, Zaidi J, Campbell S, Collin W. Blood flow changes in the ovarian and uterine arteries during the normal menstrual cycle. Am J Obstet Gynecol 1996; 175: 625-631.

9- Steer CV, Campbell S, Pampiglione JS, Kingsland CR, Mason BA, Collins WP. Transvaginal colour flow imaging of the uterine arterics during the ovarian and menstrual cycles. Hum Reprod 1990; 5: 391-395.

10- Friedler S, Schenker J, Herman A et al. The role of 
ultrasonography in the evaluation of endometrial receptivity following assisted reproductive treatments: a critical review. Hum Reprod Update 1996; 2: 323-335.

11- Steer C, Campbell S, Tan S, et al. The use of transvaginal color flow imaging after in vitro fertilization to identify optimum uterine conditions before embryo transfer. Fertil Steril 1992; 57: 372-376.

12- Sher G, Fisch J. Vaginal sildenafil (Viagra): a preliminary report of a novel method to improve uterine artery blood flow and endometrial development in patients undergoing IVF. Hum Reprod 2000; 15: 806-809.

13- Hull MG, Glazener CM, Kelly NJ, Conway DI, Foster PA, Hinton RA et al. Population study of causes, treatment and outcome of infertility. $\mathrm{Br}$ Med J 1985; 291: 1693-1697.

14- Fleischer AC, Herbert CM, Hill GA, Kepple DM, Worrell JA. Transvaginal sonography of the endometrium during induced cycles. $J$ Ultrasound Med 1991; 10: 93-95.

15- Sleer CV, Tan SL, Mason BA, Campbell S. Midluteal-phase vaginal color Doppler assessment of uterine artery impedance in a subfertile population. Fertil Steril 1994; 61: 53-58.

16- Gonen Y, Casper RF. Prediction of implantation by the sonographic appearance of the endometrium during controlled ovarian stimulation for in vitro fertilization (IVF). $\mathrm{J}$ in vitro Fertil Embryo Trans 1990; 41 : 146-152.

17- Miles RD, Menke JA, Bashiru M, Colliver JA. Relationships of five Doppler measures with flow in an in vitro model and clinical findings in newborn infants. J Ultrasound Med 1987; 6: 597-599.

18- Nakai A, Yokota A, Koshino T, Araki T. Assessment of endometrial perfusion with Doppler ultrasound in spontaneous and stimulated menstrual cycles. J Nippon Med Sch 2002; 69(4): 328-332.

19- Carbillon L, Perrot N, Uzan M, Uzan S. Doppler ultrasonography and implantation: a critical review. Fetal Diagn Ther 2001; 16(6): 327-332.

20- Isaksson R, Tiitinen A, Reinikainen LM,. Cacciatore B. Comparison of uterine and spiral artery blood flow in women with unexplained and tubal infertility. Ultrasound Obstet Gynecol 2003; 21(2): 174-180.

21- Tohma H, Hasegawa I, Sekizuka N, Tanaka K. Uterine blood flow. Assessment in an intrauterine insemination program for unexplained infertility. $J$ Reprod Med. 1997; 42(8): 463-466.

22- Czekierdowski A, Zrubek H, Bednarek W. Color and power Doppler ultrasonographic measurement of the uterine blood flow in subfertile women with unstimulated cycles. Ginekol Pol 1998; 69(12): 1191-1197.

23- Jirous J, Diejomaoh ME, AL-Abdulhadi F, Boland MH, Nazar M. A comparison of the uterine and intraovarian arterial flows in nonpregnant women having a history of recurrent spontaneous miscarriage associated with antiphospholipid syndrome. Arch Gynecol Obstet 2004; 270(2): 74-78.

24- Habara T, Nakatsuka M, Konishi H, Asagiri K, Noguchi S, Kudo T. Elevated blood flow resistance in uterine arteries of women with unexplained recurrent pregnancy loss. Hum Reprod 2002; 17(1): 190-194.

25- Nakatsuka M, Habara T, Noguchi S, Konishi $H$, Kudo T. Impaired uterine arterial blood flow in pregnant women with recurrent pregnancy loss. J Ultrasound Med 2003; 22(1): 27-31.

26- Kupesic C, Kurjak A. Uterine and ovarian perfusion during the periovulatory period assessed by transvaginal color Doppler Fertil Steril 1993; 60: 439-443.

27- Edi-Osagie EC, Seif MW, Aplin JD, Jones CJ, Wilson G, Lieberman BA. Characterizing the endometrium in unexplained and tubal factor infertility. A multiparametric investigation. Fertil Steril 2004; 82(5): 1379-1389.

28- Check JH, Gandica R, Dietterich C. Lurie D. Evaluation of a nonhomogeneous endometrial echo pattern in the midluteal phase as a potential factor associated with unexplained infertility. Fertil Steril 2003; 79(3): 590-593.

29. Salle B, Bied-Damon V, Benchaib M. Preliminary report of an ultrasonography and colour Doppler uterine score to predict receptivity in an in-vitro fertilization programme. Hum Reprod 1998; 13: 1669-1673.

30- Steer CV, Tan SL, Dillon D. Vaginal color Doppler assessment of uterine artery impedence correlates with immunohistochemical markers of endometrial receptivity required for the implantation of an embryo. Fertil Steril 1995; 63: 101-108.

31- Bassil S, Magritte JP, Roth J. Uterine vascularity during stimulation and its correlation with implantation in in-vitro fertilization. Hum Reprod 1995; 10: 1497-1501.

32- Coulam CB, Bustillo M, Soenksen DM, Britten S. Ultrasonic predictors of implantation after assisted reproduction. Fertil Steril 1994; 62: 1004-1010.

33- Yaron Y, Peyser MR, Botchan A. Endometrial receptivity in the light of modern assisted reproductive technologies. Fertil Steril 1994; 62: 225-232. 
Table I : Clinical and ultrasonographic features of patients in the studied groups.

\begin{tabular}{|c|c|c|c|c|c|}
\hline & Group (A) & Group (B) & Group (C) & $\mathbf{F}$ & $\mathbf{P}$ \\
\hline Number of patients ( $\mathbf{n}$ ) & 30 & 20 & 20 & & \\
\hline Age (years) $\mathrm{X} \pm \mathrm{SD}$ & $29.96 \pm 3.57$ & $29.05 \pm 4.55$ & $29.25 \pm 3.59$ & 0.29 & 0.43 \\
\hline $\begin{array}{l}\mathrm{PI} \\
\text { Range } \\
\mathrm{X} \pm \mathrm{SD}\end{array}$ & $\begin{array}{c}0.9-2.7 \\
1.83 \pm 0.55\end{array}$ & $\begin{array}{c}1.1-2.5 \\
1.73 \pm 0.49 \\
\end{array}$ & $\begin{array}{c}0.8-2.7 \\
1.84 \pm 0.48\end{array}$ & 0.32 & 0.72 \\
\hline $\begin{array}{l}\text { Endometrial thickness }(\mathrm{mm}) \\
\text { Range } \\
X \pm S D\end{array}$ & $\begin{array}{c}6-13 \\
9.17 \pm 1.93^{*}\end{array}$ & $\begin{array}{c}6-15 \\
9.1 \pm 2.13^{*}\end{array}$ & $\begin{array}{c}10-17 \\
12.65 \pm 2.22\end{array}$ & 20.5 & 0.001 \\
\hline
\end{tabular}

* Significant in relation to group $\mathrm{C}$

Table II : Correlation between pulsatility index of uterine artery and other factors.

\begin{tabular}{|l|c|c|}
\hline \multicolumn{1}{|c|}{ Variables } & R & P \\
\hline Age (years) & 0.466 & 0.001 \\
\hline Endometrial thickness (mm): & & \\
Group A & -0.136 & 0.393 \\
Group B & -0.26 & 0.19 \\
Group C & -0.27 & 0.098 \\
\hline
\end{tabular}

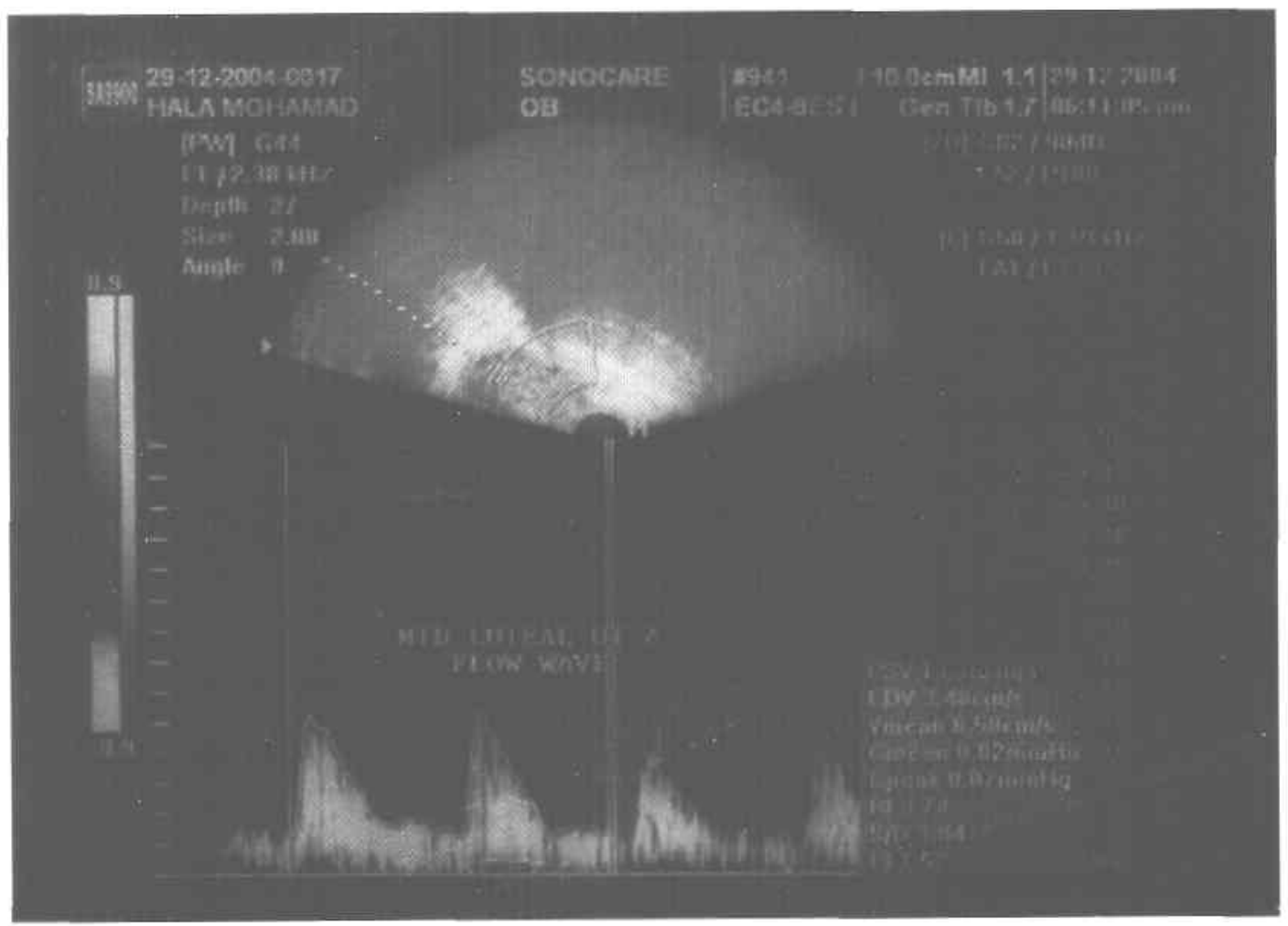

Fig. 1. An example of a flow velocity waveform during the midluteal phase of a spontaneous cycle in a normal fertile woman (pulsatility index $=1.52$ ) 


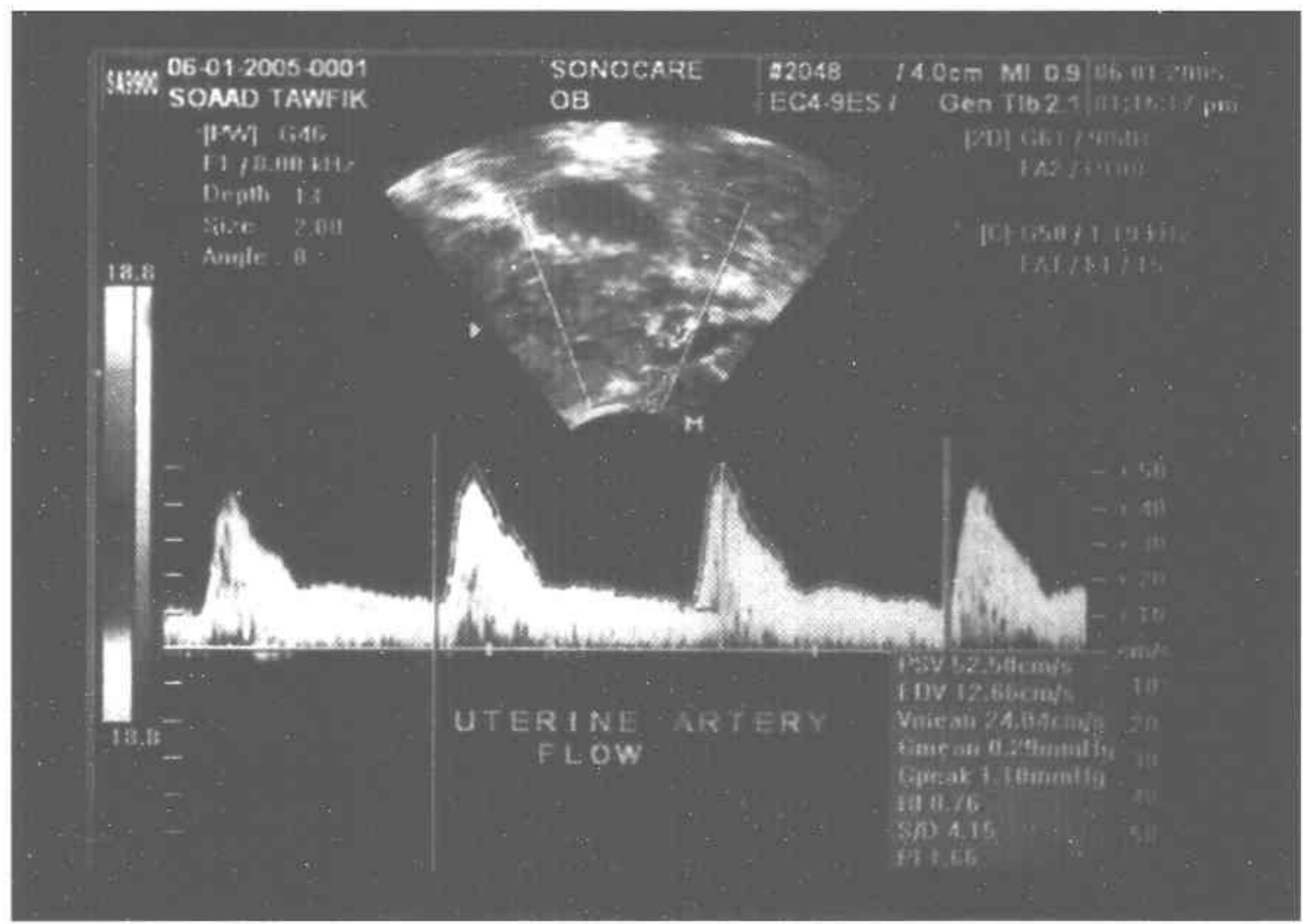

Fig. 2. An example of a flow velocity waveform during the midluteal phase of a spontaneous cycle in a woman with unexplained infertility (pulsatility index $=1.66$ ).

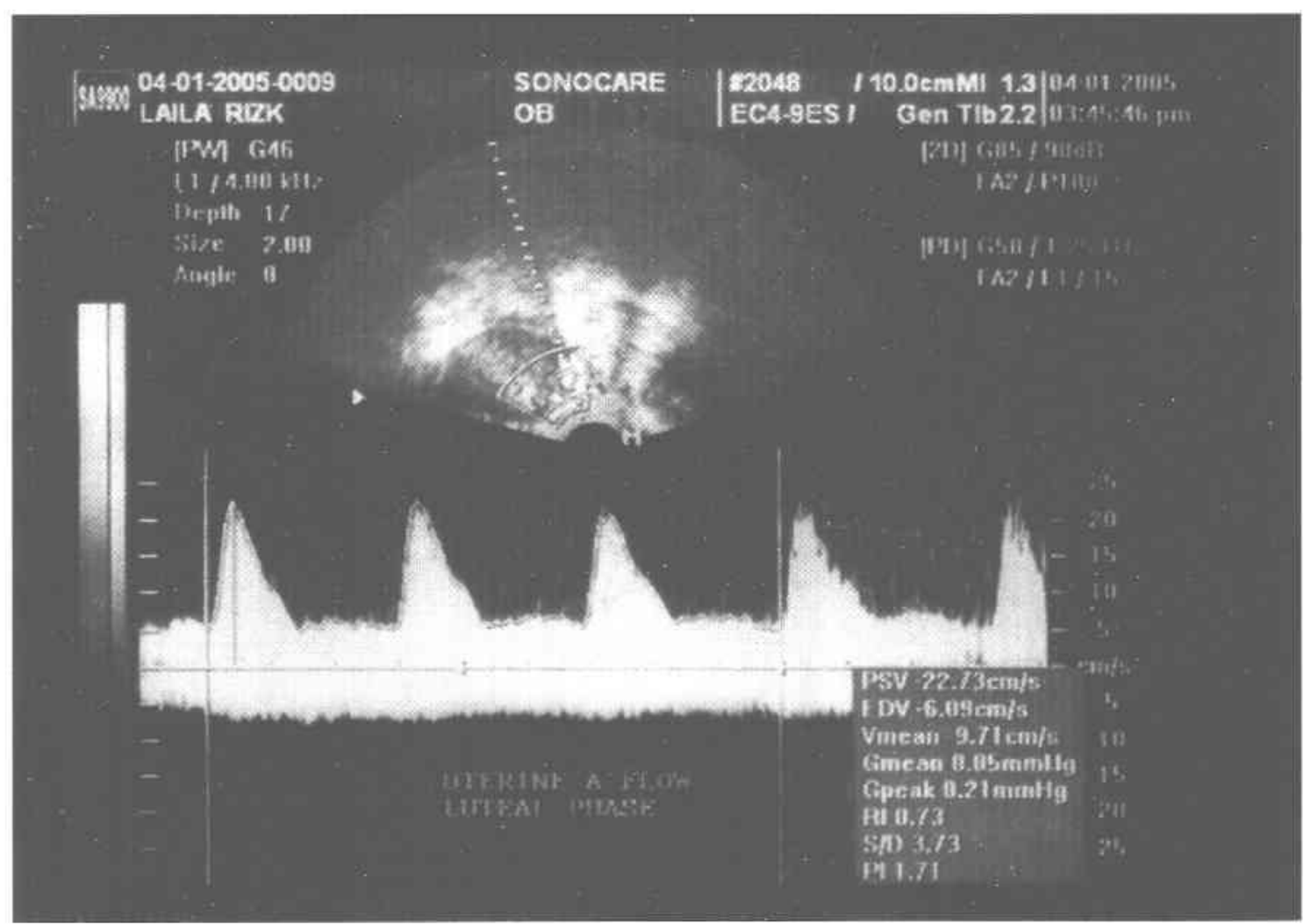

Fig. 3. An example of a flow velocity waveform during the midluteal phase of a spontaneous cycle in a woman with recurrent pregnancy loss (pulsatility index $=1.71$ ). 